\title{
Quantitative metabolomics analysis of amino acid metabolism in recombinant Pichia pastoris under different oxygen availability conditions
}

\author{
Marc Carnicer', Angela ten Pierick², Jan van Dam², Joseph J Heijnen², Joan Albiol' ${ }^{1}$, Walter van Gulik²*
} and Pau Ferrer ${ }^{1 *}$

\begin{abstract}
Background: Environmental and intrinsic stress factors can result in the global alteration of yeast physiology, as evidenced by several transcriptional studies. Hypoxia has been shown to have a beneficial effect on the expression of recombinant proteins in Pichia pastoris growing on glucose. Furthermore, transcriptional profiling analyses revealed that oxygen availability was strongly affecting ergosterol biosynthesis, central carbon metabolism and stress responses, in particular the unfolded protein response. To contribute to the better understanding of the effect and interplay of oxygen availability and foreign protein secretion on central metabolism, a first quantitative metabolomic analysis of free amino acids pools in a recombinant $P$. pastoris strain growing under different oxygen availability conditions has been performed.
\end{abstract}

Results: The values obtained indicate significant variations in the intracellular amino acid pools due to different oxygen availability conditions, showing an overall increase of their size under oxygen limitation. Notably, even while

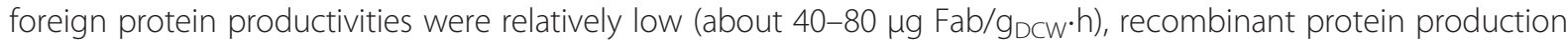
was found to have a limited but significant impact on the intracellular amino acid pools, which were generally decreased in the producing strain compared with the reference strain. However, observed changes in individual amino acids pools were not correlated with their corresponding relative abundance in the recombinant protein sequence, but to the overall cell protein amino acid compositional variations.

Conclusions: Overall, the results obtained, combined with previous transcriptomic and proteomic analyses provide a systematic metabolic fingerprint of the oxygen availability impact on recombinant protein production in P. pastoris.

Keywords: Pichia pastoris, Metabolite quantification, Recombinant protein production, Hypoxia, Amino acids, Metabolic burden

\section{Background}

Pichia pastoris has emerged as a workhorse for the production of recombinant proteins [1-4]. Moreover, the development of both synthetic and systems biotechnology tools specific for this cell factory platform [5-13], has opened new opportunities for metabolic engineering, as well as rational design and optimization of media composition and culture conditions.

\footnotetext{
*Correspondence: w.m.vangulik@tudelft.nl; pau.ferrer@uab.cat

${ }^{2}$ Department of Biotechnology, Delft University of Technology, Julianalaan 67, Delft 2628BC, The Netherlands

'Department of Chemical Engineering, Universitat Autònoma de Barcelona, Bellaterra, (Cerdanyola del Vallès) 08193, Spain
}

Recombinant protein overproduction often results in a metabolic burden. Such effect may be reflected on process parameters such maximum growth rate, biomass yield or specific substrate consumption of yeast cells [4,14-17], thus suggesting a potential impact on the cell's energy metabolism, possibly derived from higher maintenance requirements [18]. Furthermore, production of recombinant proteins may cause cellular stress due to unfolded proteins and unsuitable or inefficient secretion [19], which, in turn, may negatively affect cell growth, even at relatively low expression levels $[15,20]$, that is, at the product yields range where effects derived from increased energy and precursor demands for protein

\section{Biomed Central}


synthesis on cell growth should be theoretically negligible. In this context, limited but significant alterations in the carbon flux distribution over the central metabolism have been recently reported [21-24]. Also, amino acid supplementation of the growth medium has been shown to partially unburden cellular metabolism during recombinant protein production in yeast [23,25-27]. Interestingly, such studies in $P$. pastoris have provided novel evidence that the adaptation of the central metabolism to recombinant protein production can not only be explained by an increased drain of precursors for protein synthesis [23]. Indeed, amino acids are not only important precursors for protein synthesis but also participate in the regulation of major metabolic pathways. Glutamic and Aspartic acid for instance, are components of the aspartate/malate redox shuttle [28] and their concentrations may indirectly impact on the rate of oxidation of glycolytic NADH.

We have previously reported the beneficial impact of hypoxia conditions on recombinant protein production in P. pastoris [29]. The physiological bases of this beneficial effect were further investigated in a recent multilevel study including transciptome, proteome and metabolic flux analyses [30]. These studies allowed to gather information on the biological processes involved in the adaptation to hypoxia and their relation with extracellular recombinant protein production in P. pastoris. However, the potential effect of oxygen availability and/or recombinant protein production on the intracellular metabolite levels (particularly of the amino acid precursors used for protein synthesis) remains to be elucidated.

To investigate the potential impact of foreign protein expression and secretion on amino acid metabolism, the free intracellular amino acids pools were analyzed in carbon limited chemostat cultivations at a fixed growth rate and different oxygenation conditions, using a recombinant $P$. pastoris strain secreting an antibody Fab fragment $(\mathrm{E})$. The results are compared to the reference (non-producing) strain (C) and further combined with the metabolic fluxes related to protein synthesis and global transcriptome dataset from our previous study performed with the same strains and analogous cultivation conditions [30]. Overall, this study aimed at understanding further the behaviour of the variations in intracellular amino acid levels as a result of the different oxygenation conditions employed, as well as gaining further insight in the potential interactions between energy metabolism and amino acid metabolism and, how such interactions may be perturbed by heterologous protein secretion in $P$. pastoris.

\section{Results and discussions}

\section{Growth and recombinant protein secretion in recombinant $P$. Pastoris}

The burden caused by recombinant protein production in yeast and, in particular, $P$. pastoris, has been recently suggested to impact the central metabolism even at relatively low expression levels, i.e. where increased precursor (amino acids) demands for recombinant protein production may be negligible $[10,23,24]$. Integration of transcriptomic, fluxomic and metabolomic data of recombinant cells under different environmental conditions may help to understand the metabolic adaptations of the cell's central metabolism to protein production under different environmental conditions, particularly in relation to amino acid metabolism. In this study, intracellular amino acid pools of a recombinant $P$. pastoris strain expressing an antibody Fab fragment under the control of a constitutive pGAP promoter were measured during growth in glucose-limited chemostat cultures under different oxygen availability conditions. The macroscopic growth parameters for both the control and Fab-producing P. pastoris strains during growth at three different oxygenation levels are given in Table 1 . Coherent with previous studies $[30,31]$, the adaptation from normoxic (fully aerobic) to hypoxic conditions lead to a shift from fully respiratory to respiro-fermentative metabolism, as well as increased

Table 1 Summary of macromolecular culture parameters

\begin{tabular}{|c|c|c|c|c|c|c|}
\hline & \multicolumn{3}{|c|}{ Control Strain } & \multicolumn{3}{|c|}{ Expressing Strain } \\
\hline & Normoxic & $\mathrm{O}_{2}$-limited & Hypoxic & Normoxic & $\mathrm{O}_{2}$-limited & Hypoxic \\
\hline $\mathrm{q}_{\mathrm{Fab}}$ & & & & $40 \pm 5$ & $82 \pm 2$ & $74 \pm 9$ \\
\hline $\mathrm{q}_{\mathrm{Glc}}$ & $-1.00 \pm 0.02$ & $-1.28 \pm 0.03$ & $-1.72 \pm 0.05$ & $-1.01 \pm 0.02$ & $-1.37 \pm 0.03$ & $-1.56 \pm 0.04$ \\
\hline $\mathrm{qO}_{\mathrm{O}}$ & $-2.35 \pm 0.06$ & $-2.01 \pm 0.07$ & $-2.01 \pm 0.15$ & $-2.44 \pm 0.07$ & $-1.99 \pm 0.08$ & $-1.81 \pm 0.13$ \\
\hline qCO2 & $2.43 \pm 0.06$ & $2.55 \pm 0.06$ & $3.21 \pm 0.14$ & $2.52 \pm 0.07$ & $2.68 \pm 0.07$ & $2.94 \pm 0.12$ \\
\hline$q_{x}$ & $3.57 \pm 0.15$ & $3.83 \pm 0.18$ & $3.77 \pm 0.23$ & $3.55 \pm 0.15$ & $3.77 \pm 0.18$ & $3.58 \pm 0.22$ \\
\hline $\mathrm{q}_{\mathrm{EtOH}}$ & & $0.31 \pm 0.02$ & $0.84 \pm 0.06$ & & $0.41 \pm 0.03$ & $0.83 \pm 0.06$ \\
\hline $\mathrm{q}_{\text {Ara }}$ & & $0.13 \pm 0.01$ & $0.33 \pm 0.01$ & & $0.19 \pm 0.01$ & $0.24 \pm 0.02$ \\
\hline $\mathrm{RQ}$ & $1.03 \pm 0.04$ & $1.27 \pm 0.05$ & $1.60 \pm 0.14$ & $1.03 \pm 0.04$ & $1.34 \pm 0.06$ & $1.63 \pm 0.13$ \\
\hline
\end{tabular}

$\mathrm{q}_{\mathrm{Gluc}}$ and $\mathrm{q}_{\mathrm{O} 2}$ are specific utilization rates, and $\mathrm{q}_{\mathrm{X}}, \mathrm{q}_{\mathrm{Ara}}, \mathrm{q}_{\mathrm{EtOH}}$ and $\mathrm{q}_{\mathrm{CO} 2}$ are specific production rates, where Glc, Ara, EtOH and $\mathrm{X}$ stand for glucose, arabinitol, ethanol and biomass, respectively. Conversion rates are given in $\mathrm{mmol} /\left(\mathrm{g}_{\mathrm{DCw}} \cdot \mathrm{h}\right)$, except for $\mathrm{q}_{\mathrm{Fab}}$, which is given in $\mu \mathrm{gFab} /\left(\mathrm{g}_{\mathrm{DCw}} \cdot \mathrm{h}\right)$. DCW, $\mathrm{dry}$ cell weight. $\mathrm{RQ}=\mathrm{qCO}_{\mathrm{CO}} / \mathrm{qO}_{2}$. 
secreted recombinant product productivities. Importantly, growth parameters for the normoxic and oxygenlimiting conditions were statistically identical to those previously reported by Baumann and co-workers in a transcriptomic, proteomic and fluxomic profiling study of the same strains growing under analogous conditions [30]. Conversely, the hypoxic condition tested in this study appeared to be less stringent compared with the corresponding cultivation condition previously reported by Baumann and co-workers [30], as indicated by a lower respiratory quotient (RQ), as well as lower specific rates of ethanol and arabinitol production (See Additional file 1 for a vis-à-vis comparison of results between this study and data reported in [30]). This was further supported by the fact that, in our previous studies only 3.5 residence times could be accomplished in the hypoxic condition [29-31], whereas in this study the hypoxic condition could be extended up to a minimum of 5 residence times, which is the period required to reach a true metabolomic steady state [32].

\section{Global analysis of intracellular amino acid pools}

To obtain a global overview of the effects of different oxygen supply conditions and recombinant protein secretion on the measured intracellular free amino acid pool sizes, we subjected the relative changes in metabolite pool sizes to Principal Component Analysis (PCA) (Figure 1; see also Supplementary Material 1 for full data from PCA analysis). PCA projection demonstrated that the maximum variability in the data set clearly differentiated between different oxygenation conditions (precisely, between normoxia and the two oxygen-restricted conditions), with the first component (PC1) covering $71.3 \%$ of the data variance. The second principal component (PC2), which explained only $19.2 \%$ of the total amino acid pools variance, clearly discriminated between the Fab expressing and the reference strain, indicating a limited impact of the antibody fragment production on the P. pastoris amino acid metabolome. Ala, Trp and Asp were the amino acids with the highest contribution $(24.0 \%, 20.6 \%$ and $19.7 \%$ respectively) to the variance in $\mathrm{PC} 2$.

Overall, these data reflect a higher impact of oxygen availability rather than recombinant protein production on the global physiologic response of P. pastoris, consistent with previous transcriptomic, proteomic and metabolic flux analyses [30]. To help decipher the potential dependence of the amino acid metabolism on oxygen availability and the burden caused by recombinant protein secretion, a more detailed analysis was performed focusing on each of these two factors separately.

\section{Recombinant protein production effect}

The amino acid pools provide building blocks for protein synthesis. Therefore, an impact of Fab production on these metabolites was a priori expected. In fact, previous studies using amino acids for media supplementation or complex extracts have proven to have a positive effect on recombinant protein production $[22,27,33]$.

In order to analyze more specifically the potential effects of recombinant protein production on the $P$. pastoris amino acid metabolite levels, a comparison was made of the free amino acid pools of the reference and the Fab producing strains grown at each oxygenation condition. The metabolites levels measured in the control strain grown at each oxygenation condition were taken as reference values to be compared with the metabolites of the Fab-expressing strain grown at the corresponding condition, thereby obtaining concentration ratios for each metabolite of the Fab-expressing relative to the control strain at each culture condition (Figure 2). In addition, two-tailed $T$-test statistical analyses allowed to identify those metabolite ratios that were significantly higher or lower than 1 (that is, those metabolite pool sizes that varied significantly between both strains).

The impact of recombinant protein production on amino acid pool sizes under normoxic conditions is shown in Figure 2A. Overall, 10 out of 17 amino acid pools increased their intracellular levels significantly, while only 2 of them had an opposite behavior. In particular, most of the free amino acids from the Ala, Asp

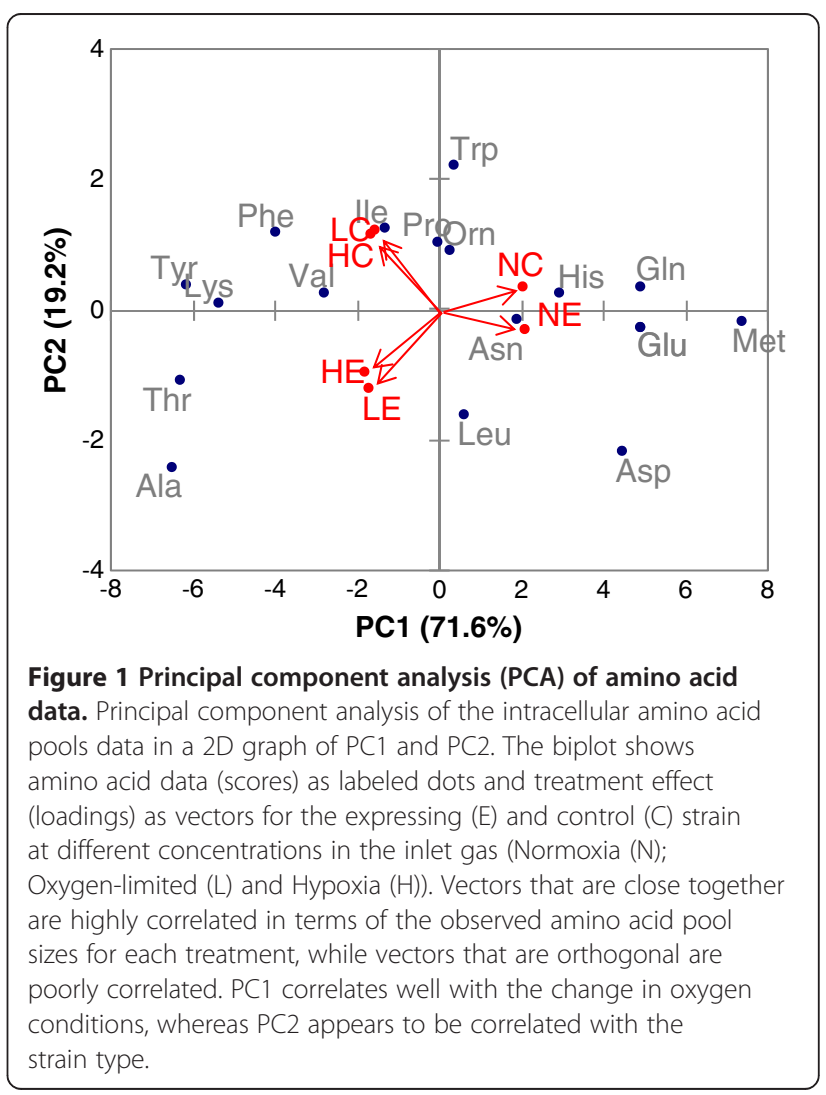



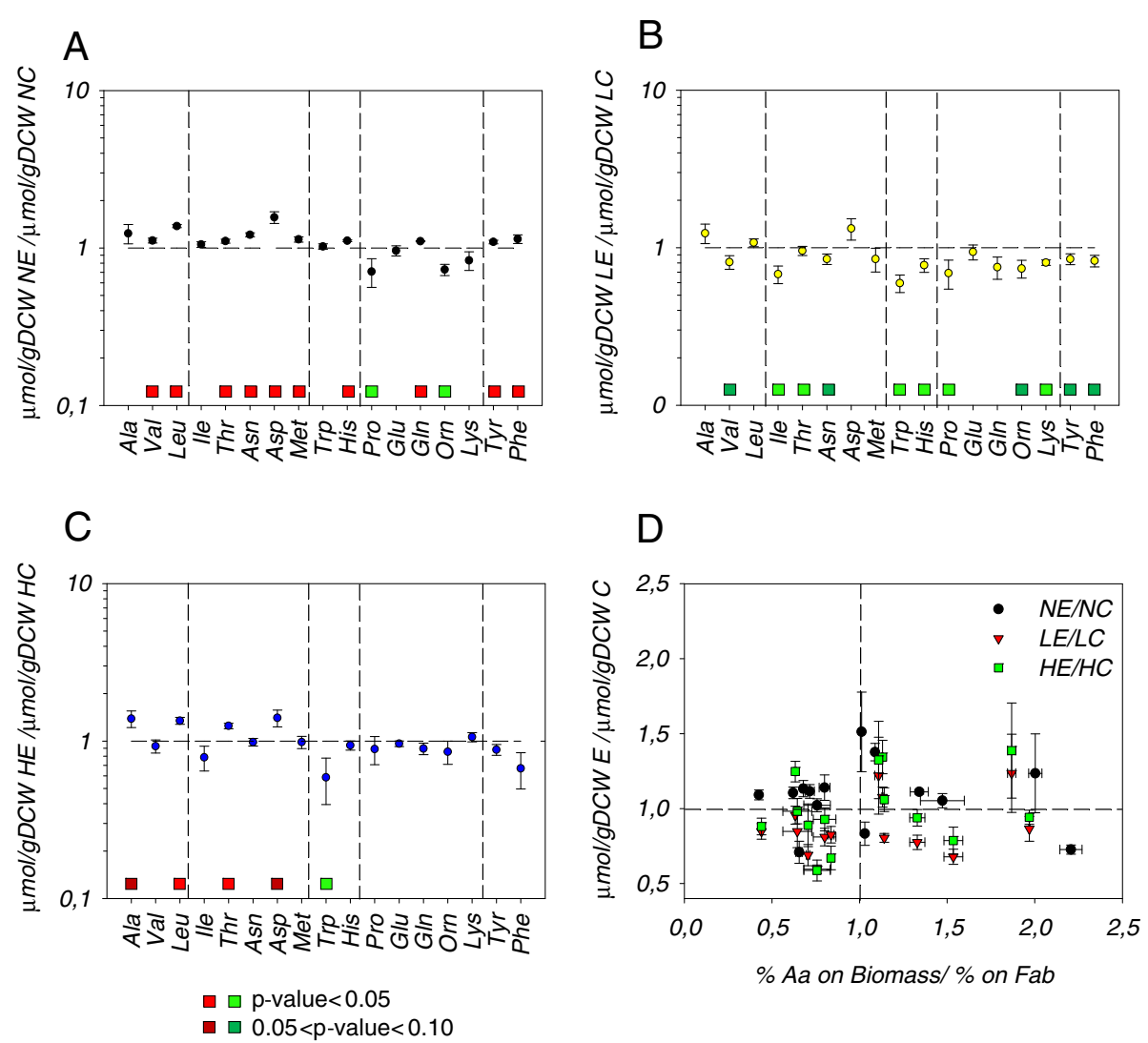

Figure 2 Amino acid pools comparison of $P$. pastoris control (C) and Fab expressing (E) strains under different oxygen conditions. The values are the average and the standard error of calculated ratios. Ratio errors were calculated using error propagation. Horizontal dashed line represents a ratio of 1 . In A, B and C vertical dashed line separate metabolites belonging to the same metabolic pathway. The squares over the metabolite names denote the results from the T-Student test. Green and red colors indicate lower and higher ratios respectively. A: normoxic condition; B: $\mathrm{O}_{2}$-limited condition; C: hypoxic conditions. D: Biomass/Fab amino acid proportion ratios influence on the intracellular amino acid pool changes. N: Normoxic, L: $\mathrm{O}_{2}$-limited; H: Hypoxic.

and Phe families displayed significantly increased levels in the Fab-expressing strain. Nevertheless, the Glu family showed different trends depending on the amino acid, being Orn and Pro significantly decreased, while Gln increased. Increased amino acid pools in the Fab-expressing strain were not accompanied by any significant flux redistribution though the central metabolism nor proteome compositional change, when compared to the control strain growing under the same oxygenation condition [30,31].

Conversely, generally decreased amino acid pool ratios (11 out of 17) were found in the Fab-expressing strains under oxygen-limiting conditions, compared to the reference strain (Figure 2B), that is, an opposite behavior to that observed under normoxic conditions. We observed the same trend when comparing the Fab-expressing strain growing under hypoxic condition; however, the data variance was higher and, therefore, the observed changes were not statistically significant. A priori, this observation might be associated to the increased specific heterologous protein productivity found under oxygen limitation, as the Fab producing strain might require higher metabolic fluxes of amino acids for protein synthesis, thereby causing a drain of precursor pools. Nevertheless, this hypothesis is highly unlikely, since Fab production levels were relatively low in relation to the total cell protein [31]. Besides, this trend was not uniform within each of the amino acid families. For instance, in the Ala family, Ala and Leu pools increased while Val decreased in the Fabexpressing strain. Also, in the Asp amino acid family only Asp levels varied significantly. Moreover, no correlation was found between the relative abundance of each amino acid in the Fab antibody fragment and the observed changes in the corresponding free amino acid pools (Figure 2D). Nevertheless the specific Fab production correlated inversely (correlation value of -0.93 ) with the average of the metabolite ratios between Fab-expressing and reference strains at each culture condition. This may reflect a global re-adjustment of the free amino acid pools to compensate for the recombinant protein overproduction. Although such readjustment could not be 
the result from a direct drain of building blocks at higher Fab synthesis levels, other phenomena related with recombinant protein production might provide some explanations. For instance, recent studies on recombinant protein secretion using ${ }^{34} \mathrm{~S}$ labeling strategies with the same strain used in this study have revealed that about $58 \%$ of the Fab protein produced intracellularly is actually degraded within the cell, and only $35 \%$ is secreted [34]. In addition to protein degradation, increased energetic demands related with the cost of the folding, refolding and secretion processes of the Fab product could result in an overall readjustment of amino acid metabolism. In fact, when the Fab producing strain was cultivated at lower temperatures $\left(20^{\circ} \mathrm{C}\right)$, the unfolded protein stress response was reduced, leading to a reduced metabolic burden and higher specific productivities compared with cells grown at $25^{\circ} \mathrm{C}$ and $30^{\circ} \mathrm{C}$, in which an increase in energy demand was evidenced by an up-regulation of the TCA cycle, slightly higher in Fabsecreting strains [10]. Besides, increased maintenance requirements associated with heterologous protein production (e.g. unfolded protein stress response) may cause additional energy demands. Interestingly, recent amino acid supplementation studies using a recombinant $P$. pastoris strain revealed that such supplementation partially relieved the metabolic burden from recombinant protein production. Furthermore, de novo amino acid synthesis in cells growing on different combinations of amino acids supplementations was inversely correlated with the corresponding energetic cost for most amino acids [23].
The potential dependence between fold changes observed amongst amino acid pools when comparing the Fab-expressing to the reference strain and their corresponding energy and redox costs were analyzed (Figure 3). Interestingly, lower fold changes in amino acid pools with the highest energy cost (that is, aromatic amino acids family) was found under oxygen reduced conditions (that is, under higher Fab productivities), but not under normoxia. This might point at the hypothesis that the cell adjusts its overall amino acid metabolism to minimize the energetic burden caused by Fab production. Nevertheless, no trend was observed regarding the $\mathrm{C}$-mol degree of reduction of each amino acid (Figure 3).

\section{Oxygen availability effect}

Previous transcriptomic studies revealed amino acid metabolism as one of the major cellular processes regulated by oxygen availability [30]. Interestingly, the number of genes in the gene ontology group of amino acid metabolism that were down regulated under hypoxic conditions was substantially higher in the Fab-producing strain.

To investigate the potential correlation between observed changes in free amino acid pools and proteome amino acid composition upon oxygen availability changes, we related the observed fold changes for each free amino acid pool with the change in the relative abundance of the corresponding amino acid in the cell's proteome amino acid composition (Figure 4). The effect of oxygen
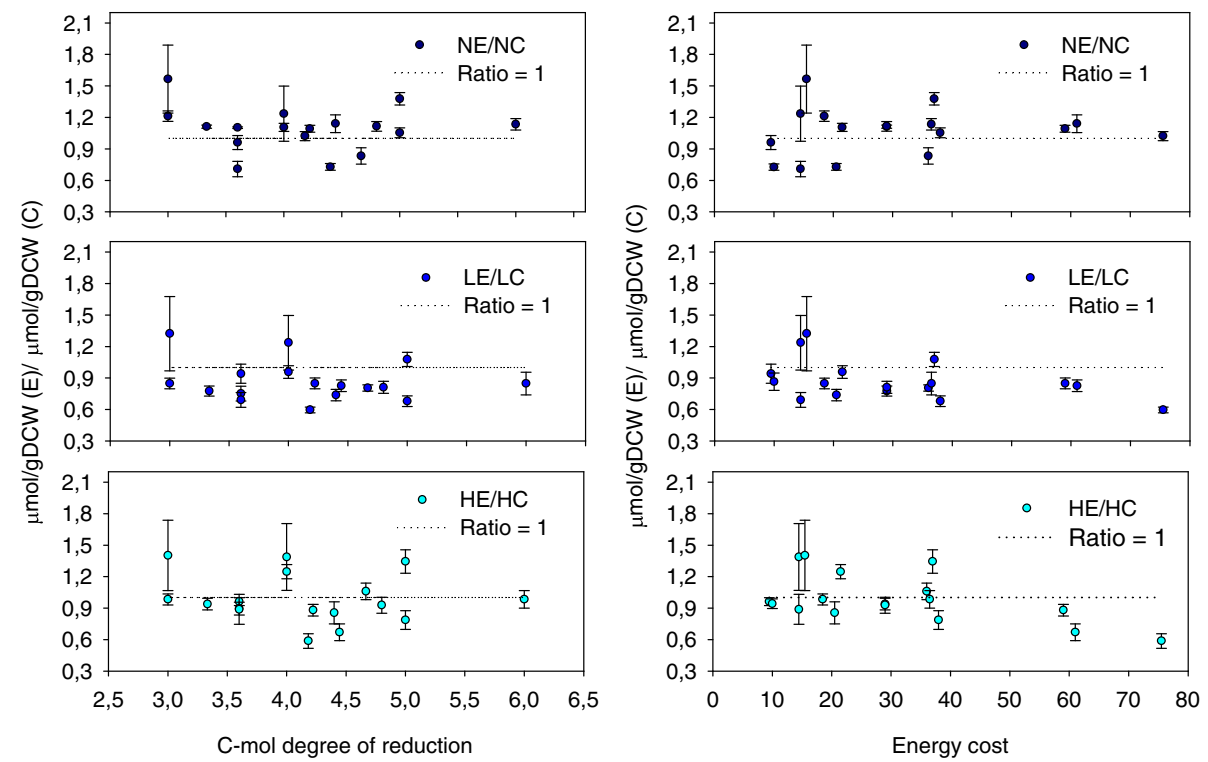

Figure 3 Amino acid C-mol degree of reduction and energy costs influence on the expressing/control amino acid pool ratios.

The values are the average and the standard error of calculated ratios. Horizontal dashed line represents a ratio of 1. Ratio errors were calculated using error propagation. The energy cost for an amino acid is defined as the number of high energy phosphate bounds $\left(\sim \mathrm{PO}_{4}\right)$ that are required for its synthesis. The amino acids energy costs were taken from S. cereviciae published data [35]. The C-mol degree of reduction of each amino acid was calculated from each molecular formula. 
availability on the cell's amino acid metabolism could be already inferred from previous measurements of the cell's protein amino acid composition [31]. For instance, the relative abundance of amino acids derived from cytosolic oxalocetate (OAA), pyruvate (Pyr), phosphoenolpyruvate and 3-phospho glycerate (3PG) were increased and those derived from mitochondrial $\alpha$-ketoglutarate $(\alpha K G)$ were reduced under hypoxic conditions. Remarkably, such changes in amino acid pools synthesized from glycolytic and tricarboxylic acid (TCA) cycle precursors appeared to be directly correlated with increased glycolytic and decreased oxidative TCA cycle fluxes under reduced oxygen levels, respectively [30,31].

Overall, changes in free amino acid pools seemed to correlate directly with changes in the relative abundance in the cell proteome for most amino acids, suggesting that biosynthetic fluxes to cell protein had a direct impact on the precursor amino acids pools sizes. A clear exception to this observation was Ala and, to a much lesser extent, Asx (Asp + Asn). Unfortunately, the available metabolomic methodology [32] did not allow for accurate quantification of the metabolite precursors of these amino acids (that is, Pyr and OAA), hampering the interpretation of the observed changes in Ala and Asx pool sizes.

To obtain an overview of the potential correlations between transcriptional changes in amino acid biosynthetic genes and intracellular amino acid levels (free pools as well as proteome amino acids) upon a change in oxygen availability, transcriptomic, metabolomic and cell protein compositional data were integrated into biosynthetic pathway maps (Figures 5 and 6; Additional file 1). The

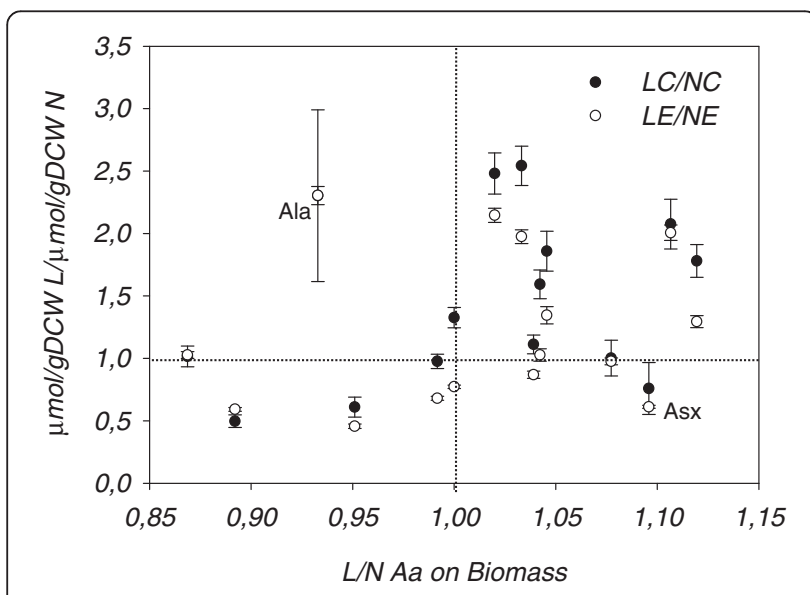

Figure 4 Impact of the differential biomass demand over the intracellular amino acid pools under different oxygen conditions. The values are the average and the standard error of calculated ratios. Horizontal and vertical dashed lines divided the graph in four spaces to facilitate the interpretation. The biomass demands under normoxia and oxygen-limited conditions were taken from [31]. Asx = Asp + Asn. specific amino acid composition of the whole protein extracts at different oxygen conditions was included in order to show the biosynthetic demand (i.e. carbon flux) for each specific amino acid synthesis pathway. Since hypoxic condition of this study was not equivalent to that used in previous transcriptomic studies, only normoxic and oxygen limiting conditions were compared for both $\mathrm{E}$ and $\mathrm{C}$ strains.

A direct comparison of the relative changes observed in the free amino acid levels between different oxygen availability conditions and the corresponding changes observed in the whole cell's amino acid component (that is, free intracellular amino acid pools plus cell protein amino acids) indicated that the latter were less pronounced.

The integrated data seem to reflect the oxygendependent transcriptional regulation of amino acid biosynthesis pathways. In particular, transcriptional levels of several key regulatory enzymes in the biosynthetic pathways correlated inversely with the carbon flow through them, as well as with their corresponding end-metabolite levels, probably denoting the negative feedback control of the pathway. For instance, the levels of free tyrosine and phenylalanine were substantially increased under oxygen-limiting conditions, concomitantly with a reduction of the mRNA levels of $A R O 7$, while an opposite pattern was observed for the free tryptophan pools and TRP3 transcriptional levels (Figure 5). This pattern was also observed in other amino acid biosynthetic pathways such as methionine (see Additional file 2).

Similarly, in the glutamate amino acid family (Figure 6), the carbon flow to lysine was increased under oxygen limiting conditions, concomitantly with a reduction in transcript levels of several genes of its pathway, particularly LYS20, the first reaction of the pathway. In addition, the carbon flux to the Glu and Gln biosynthetic pathway was significantly reduced at lower oxygen availability. This pattern correlated directly with the reduced transcriptional levels of GDH3 and GLN1.

Conversely, the reduced Ala and Asp biosynthetic fluxes to cell protein under limited oxygen levels seemed to be directly correlated to ATL1 and ATT1/ATT2 transcriptional levels, respectively (Additional files 2 and 3). Also, no significant variation on the His biosynthetic flux was observed despite the significant transcriptional and His pool sizes changes measured upon oxygen limitation.

\section{Conclusions}

Previous transcriptomic studies [30] pointed at the impact of oxygen availability and recombinant protein production on amino acid metabolism. The analysis at the metabolomic level further confirms such impact. In particular, our data point at a major impact of oxygen 


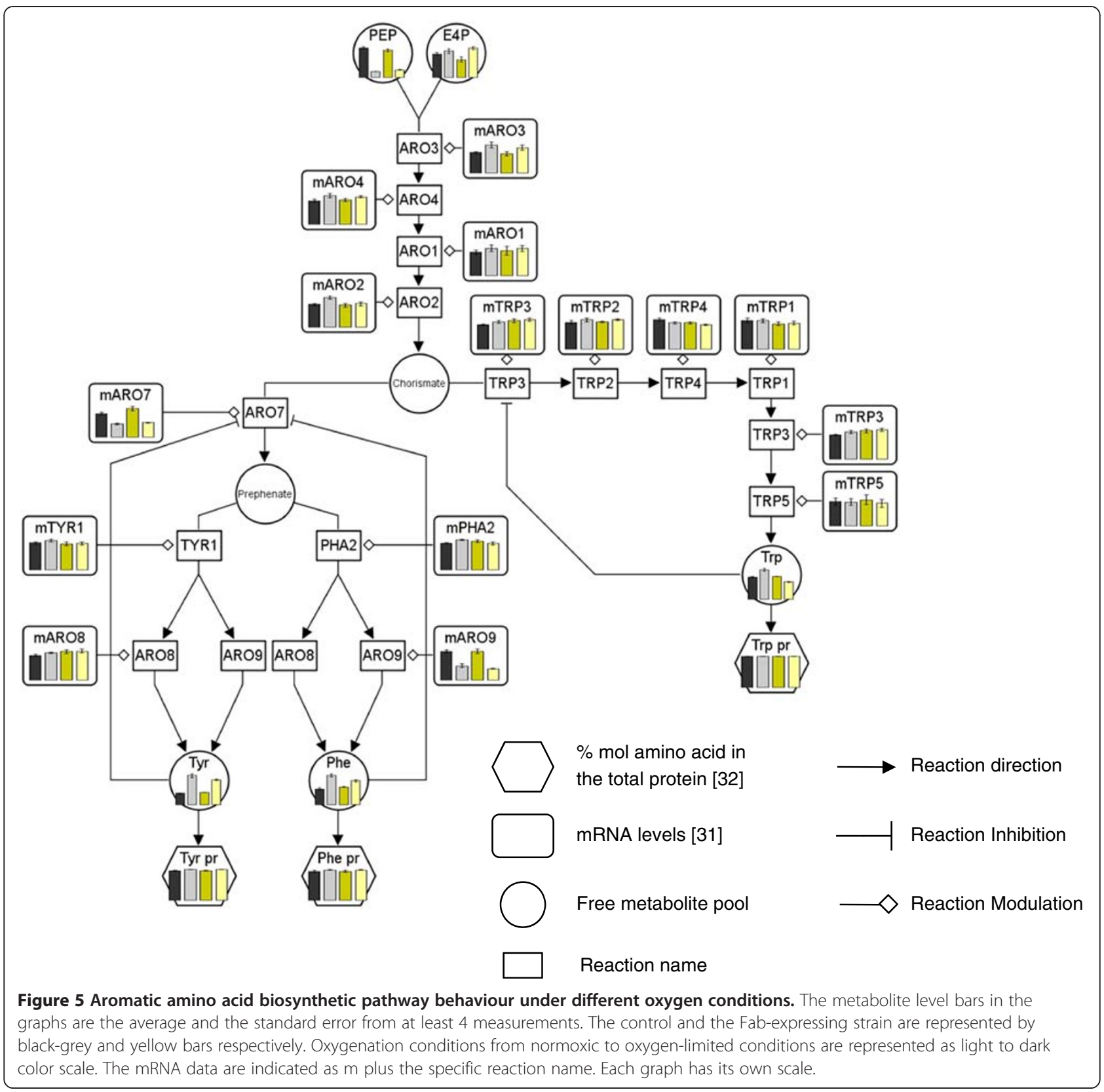

availability rather than recombinant protein production on the free amino acid pools, coherent with previous transcriptional analyses. Notably, changes in free amino acid pools observed at different oxygenation conditions generally correlated directly with the changes in relative abundances in the corresponding amino acids of the cell's proteome, with alanine being the major exception. In contrast, the impact of recombinant protein production on the free amino acids pools depended on the oxygenation state. Importantly, the observed changes did not correlate with the difference in amino acid composition of the recombinant product and the cell's proteome, but rather to the energetic costs (specifically, for those amino acids with highest energy costs), thereby suggesting a possible dependence between mitochondrial metabolism and amino acid anabolism as a potential target to modulate the metabolic burden caused by recombinant protein production.

Systematic integration of metabolomic and transcriptional data into genomic-scale metabolic models should allow gaining further understanding of the behaviour of central and amino acid metabolism, as well as identifying metabolic bottlenecks limiting enhanced recombinant protein production. 


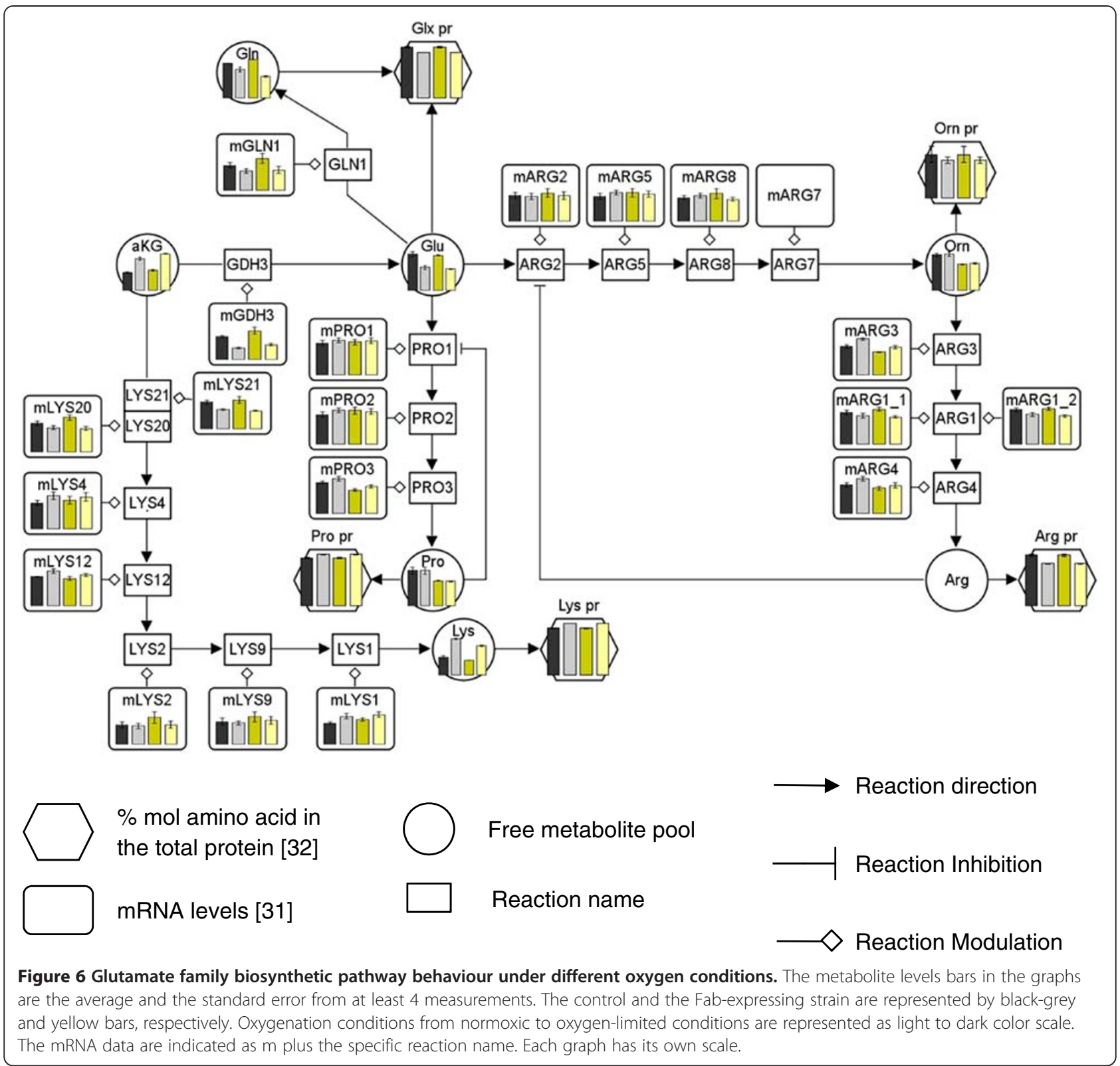

\section{Methods}

\section{Strain and cultivation conditions}

Analytical grade reagents were supplied by SigmaAldrich. HPLC-grade methanol and ethanol were supplied by J.T. Baker.

In this study, the $P$. pastoris strain X-33 pGAPZ $\alpha A$ Fab3H6 [36], secreting the light and heavy chains of a human monoclonal antibody Fab fragment under the constitutive GAP promoter and the S. cerevisiae alphamating factor leader, was used as expressing strain. A strain with an integrated empty-vector was used as reference strain. The experimental set up was as described in [32]. Briefly, glucose-limited chemostat cultures at a dilution rate of $0.1 \mathrm{~h}^{-1}$ at different oxygenation conditions were carried out by changing the oxygen content of the inlet gas. Initially, the oxygen concentration in the inlet gas stream corresponded to normal air $(20.95 \% \mathrm{v} / \mathrm{v})$ leading to a totally normoxic condition (i.e. $\mathrm{pO}_{2}>20 \%$, fully respiratory metabolism). Inlet gas oxygen levels were subsequently stepwise reduced by replacing different air proportions with nitrogen. Thereby either oxygen limited or hypoxic conditions were created in the bioreactor which are characterized by different ethanol and arabinitol production rates [29-31]. Two chemostats were performed for each metabolic steady state and strain. 


\section{Sampling}

The different chemostat conditions were maintained for 5 residence times before sampling. As previously reported, this cultivation time is enough to reach a metabolic steady state in $P$. pastoris [32]. For each steady state condition, duplicate samples for intracellular metabolite measurement were taken using the previously described optimized protocol for the direct measurement of $P$. pastoris metabolome [32]. For cellular dry weight, a known volume of cultivation broth was filtered using pre-weighted filters; these were washed with two volumes of distilled water and dried to constant weight at $70^{\circ} \mathrm{C}$

Samples for extracellular metabolite were obtained by rapid sampling of broth with immediate cooling to $0^{\circ} \mathrm{C}$ and fast filtration, using the cold steel-bead method [37] and analyzed using high-performance liquid chromatography (HPLC) with a Bio-Rad Aminex column at a temperature of $60^{\circ} \mathrm{C}$. The mobile phase was $5.0 \mathrm{mM}$ phosphoric acid of which the flow rate was set to $0.6 \mathrm{~mL} / \mathrm{min}$.

\section{Free intracellular amino acid analysis}

The intracellular concentrations of Ala, Val, Leu, Ile, Thr, Asn, Asp, Met, Trp, His, Pro, Glu, Gln, Orn, Lys, Tyr and Phe were determined by GC-MS [38]. Briefly, $100 \mu \mathrm{L}$ of sample were transferred to a glass vial, $30 \mu \mathrm{L}$ of $100 \mathrm{mg} / \mathrm{mL} \mathrm{NaCl}$ were added and the mixture was lyophilized. $75 \mu \mathrm{L}$ acetonitrile and $75 \mu \mathrm{L}$ of N-methyl-N -(tert- butyldimethylsilyl)trifluoroacetamide (MTBSTFA, Thermo Scientific) were added and the vial was incubated for $1 \mathrm{~h}$ at $70^{\circ} \mathrm{C}$. Subsequently, the sample was centrifuged $(10,000 \mathrm{~g}, 2 \mathrm{~min})$ and $60 \mu \mathrm{L}$ of the supernatant were transferred to a GC glass vial with an insert. The sample was then analyzed by GC-MS instrument coupled to a 5975 C MSD single quadrupole mass spectrometer (Agilent, Santa Clara, CA, USA). Glycine levels were also measured in this analysis; however, the obtained results were not included as the values were found to be inconsistent [32]. Quantification was based on isotope dilution mass spectrometry (IDMS) [39]. In total, 17 amino acids were analyzed plus Glycine.

In order to graphically visualize the obtained intracellular quantification together with the central carbon metabolism and amino acid biosynthetic pathways the VANTED software was used [40].

\section{Antibody fragment quantification}

Fab amounts in soluble cell extracts and in culture broths were performed by means of a sandwich ELISA assay as previously described [29].

\section{Principal component analysis}

Reference values for each amino acid pool level were calculated as mean of all measurements independently of the strain or oxygen availability. Afterwards, for each condition, the specific amino acid measurements were divided by the reference value to obtain a free scale value of its variation. These relative fold changes for each condition were illustrated through PCA using the xlstat plug-in software for Excel.

\section{Determination of energy and degree of reduction for amino acid synthesis}

The degree of reduction of each amino acid were calculated using the amino acid molecular formulas as explained elsewhere [41]. Energy costs for the biosynthesis of each amino acid were taken from previously published data for $S$. cerevisiae growing under aerobic conditions [35] as both yeast share equal amino acid biosynthetic pathways $[7,8,11]$.

\section{Additional files}

\section{Additional file 1: Comparison of physiological parameters of} chemostat cultivations.

Additional file 2: Alanine and serine families' biosynthetic pathways behaviour under different oxygen conditions.

Additional file 3: Aspartate and histidine amino acid families' biosynthetic pathways behavior under different oxygen conditions.

\section{Competing interests}

The authors declare that they have no competing interests.

\section{Authors' contributions}

MC performed bioreactor cultivations, experimental data acquisition, data calculation, analysis and interpretation of results and participated in drafting the manuscript. AP and JD performed the GC-MS analysis. JA participated in the overall conceptual and experimental design of this study, interpretation of results and in drafting the manuscript. JH and WG participated in the overall conceptual and experimental design of this study, interpretation of results and in revision of the manuscript. PF participated in the overall conceptual and experimental design of this study, interpretation of results and in drafting the manuscript. All the authors read and approved the final manuscript.

\section{Acknowledgements}

This work has been supported by the Spanish program on Chemical Process Technologies (project CTQ2010-15131) of the Spanish Ministry of Science and Innovation, the Generalitat de Catalunya (Contract Grant 2009-SGR-281 and Xarxa de Referència en Biotecnologia) and the Spanish Ministry scholarship TME2009-00591.

Received: 23 February 2012 Accepted: 22 May 2012

Published: 15 June 2012

\section{References}

1. Macauley-Patrick S, Fazenda ML, McNeil B, Harvey LM: Heterologous protein production using the Pichia pastoris expression system. Yeast 2005, 22:249-270.

2. Bollok M, Resina D, Valero F, Ferrer P: Recent patents on the Pichia pastoris expression system: expanding the toolbox for recombinant protein production. Recent Pat Biotechnol 2009, 3:192-201.

3. Mattanovich D, Branduardi P, Dato L, Gasser B, Sauer M, Porro D: Recombinant protein production in yeasts. Methods Mol Biol 2012, 824:329-358.

4. Cos O, Ramón R, Montesinos UL, Valero F: Operational strategies, monitoring and control of heterologous protein production in the methylotrophic yeast Pichia pastoris under different promoters: a review. Microb Cell Fact 2006, 5:17. 
5. De Schutter $K$, Lin $Y$-C, Tiels $P$, Van Hecke A, Glinka S, Weber-Lehmann J, Rouzé P, Van de Peer Y, Callewaert N: Genome sequence of the recombinant protein production host Pichia pastoris. Nat Biotechnol 2009, 27:561-566.

6. Mattanovich D, Graf AB, Stadlmann J, Dragosits M, Redl A, Maurer M, Kleinheinz M, Sauer M, Altmann F, Gasser B: Genome, secretome and glucose transport highlight unique features of the protein production host Pichia pastoris. Microb Cell Fact 2009, 8:29.

7. Sohn SB, Graf AB, Kim TY, Gasser B, Maurer M, Ferrer P, Mattanovich D, Lee SY: Genome-scale metabolic model of methylotrophic yeast Pichia pastoris and its use for in silico analysis of heterologous protein production. Biotechnol J 2010, 5:705-715.

8. Chung BKS, Selvarasu S, Camattari A, Ryu J, Lee H, Ahn J, Lee H, Lee D-Y: Genome-scle metabolic reconstruction and in silico analysis of methylotrophic yeast Pichia pastoris for strain improvement. Microb Cell Fact 2010, 9:50-64.

9. Graf AB, Dragosits M, Gasser B, Mattanovich D: Yeast systems biotechnology for the production of heterologous proteins. FEMS Yeast Res 2009, 9:335-348.

10. Dragosits M, Stadlmann J, Albiol J, Baumann K, Maurer M, Gasser B, Sauer M, Altmann F, Ferrer $P$, Mattanovich D: The effect of temperature on the proteome of recombinant Pichia pastoris. J Proteome Res 2009, 8:1380-1392.

11. Solà A, Maaheimo H, Ylönen K, Ferrer P, Szyperski T: Amino acid biosynthesis and metabolic flux profiling of Pichia pastoris. Eur J Biocherm 2004, 271:2462-2470.

12. Solà A, Jouhten $P$, Maaheimo $H$, Sánchez-Ferrando F, Szyperski T, Ferrer P: Metabolic flux profiling of Pichia pastoris growth on glycerol/methanol mixtures in chemostat cultures at low and high dilution rates. Microbiology 2007, 153:281-290.

13. Cregg JM, Tolstorukov I, Kusari A, Sunga J, Chappell T: Expression in the yeast Pichia pastoris. Methods Enzymol 2009, 463:169-189.

14. Görgens JF, van Zyl WH, Knoetze JH, Hahn-Hägerdal B: The metabolic burden of the PGK1 and ADH2 promoter systems for heterologous xylanase production by Saccharomyces cerevisiae in defined medium. Biotechnol Bioeng 2001, 73:238-245.

15. Cos O, Serrano A, Montesinos JL, Ferrer P, Cregg JM, Valero F: Combined effect of the methanol utilization (Mut) phenotype and gene dosage on recombinant protein production in Pichia pastoris fed-batch cultures. J Biotechnol 2005, 116:321-335.

16. Vigentini I, Brambilla L, Branduardi P, Merico A, Porro D, Compagno C: Heterologous protein production in Zygosaccharomyces bailii: physiological effects and fermentative strategies. FEMS Yeast Res 2005 5:647-652.

17. Krogh AM, Beck V, Christensen LH, Henriksen CM, Møller K, Olsson L: Adaptation of Saccharomyces cerevisiae expressing a heterologous protein. J Biotechnol 2008, 137:28-33.

18. Hensing MC, Rouwenhorst RJ, Heijnen JJ, van Dijken JP, Pronk JT: Physiological and technological aspects of large-scale heterologousprotein production with yeasts. Antonie Van Leeuwenhoek 1995, 67:261-279.

19. Gasser $B$, Saloheimo M, Rinas $U$, Dragosits $M$, Rodríguez-Carmona $E$, Baumann K, Giuliani M, Parrilli E, Branduardi P, Lang C, Porro D, Ferrer P, Tutino ML, Mattanovich D: Protein folding and conformational stress in microbial cells producing recombinant proteins: a host comparative overview. Microb Cell Fact 2008, 7:11.

20. Resina D, Bollók M, Khatri NK, Valero F, Neubauer $P$, Ferrer $P$ : Transcriptional response of $P$. pastoris in fed-batch cultivations to Rhizopus oryzae lipase production reveals UPR induction. Microb Cell Fact 2007, 6:21.

21. Çelik E, Çalik P, Oliver SG: A structured kinetic model for recombinant protein production by Mut ${ }^{+}$Pichia pastoris. Chem Eng Sci 2009, 64:5028-5035.

22. Heyland J, Fu J, Blank LM, Schmid A: Quantitative physiology of Pichia pastoris during glucose-limited high-cell density fed-batch cultivation for recombinant protein production. Biotechnol Bioeng 2010, 107:357-368

23. Heyland J, Fu J, Blank LM, Schmid A: Carbon metabolism limits recombinant protein production in Pichia pastoris. Biotechnol Bioeng 2011, 108:1942-1953.
24. Jordà J, Jouhten P, Cámara E, Maaheimo H, Albiol J, Ferrer P: Metabolic flux profiling of recombinant protein secreting Pichia pastoris growing on glucose:methanol mixtures. Microb Cell Fact 2012, 8:57.

25. Görgens JF, van Zyl WH, Knoetze JH, Hahn-Hägerdal B: Amino acid supplementation improves heterologous protein production by Saccharomyces cerevisiae in defined medium. Appl Microbiol Biotechnol 2005, 67:684-691.

26. Görgens JF, Passoth V, van Zyl WH, Knoetze JH, Hahn-Hägerdal B: Amino acid supplementation, controlled oxygen limitation and sequential double induction improves heterologous xylanase production by Pichia stipitis. FEMS Yeast Res 2005, 5:677-683.

27. Hahn-Hägerdal B, Karhumaa K, Larsson CU, Gorwa-Grauslund M, Görgens J, van Zyl WH: Role of cultivation media in the development of yeast strains for large scale industrial use. Microb Cell Fact 2005 4:31.

28. Bakker BM, Overkamp KM, van Maris AJA, Kötter P, Kötter P, Luttik MaH, van Dijken JP, Pronk JT: Stoichiometry and compartmentation of NADH metabolism in Saccharomyces cerevisiae. FEMS Microbiol Rev 2001, 25:15-37.

29. Baumann K, Maurer M, Dragosits M, Cos O, Ferrer P, Mattanovich D: Hypoxic fed-batch cultivation of Pichia pastoris increases specific and volumetric productivity of recombinant proteins. Biotechnol Bioeng 2008, 100:177-183.

30. Baumann K, Carnicer M, Dragosits M, Graf AB, Stadlmann J, Jouhten P, Maaheimo H, Gasser B, Albiol J, Mattanovich D, Ferrer P: A multi-level study of recombinant Pichia pastoris in different oxygen conditions. BMC Syst Biol 2010, 4:141.

31. Carnicer M, Baumann K, Töplitz I, Sánchez-Ferrando F, Mattanovich D, Ferrer P, Albiol J: Macromolecular and elemental composition analysis and extracellular metabolite balances of Pichia pastoris growing at different oxygen levels. Microb Cell Fact 2009, 8:65-78.

32. Carnicer M, Canelas AB, Pierick A, Zeng Z, Dam J, Albiol J, Ferrer P, Heijnen JJ, Gulik W: Development of quantitative metabolomics for Pichia pastoris. Metabolomics 2012, 8:284-298.

33. Görgens JF, Planas J, van Zyl WH, Knoetze JH, Hahn-Hägerdal B: Comparison of three expression systems for heterologous xylanase production by $S$. cerevisiae in defined medium. Yeast 2004, 21:1205-1217.

34. Pfeffer M, Maurer M, Köllensperger G, Hann S, Graf AB, Mattanovich D: Modeling and measuring intracellular fluxes of secreted recombinant protein in Pichia pastoris with a novel $34 \mathrm{~S}$ labeling procedure. Microb Cell Fact 2011, 10:47.

35. Raiford DW, Heizer EM, Miller RV, Akashi H, Raymer ML, Krane DE: Do amino acid biosynthetic costs constrain protein evolution in Saccharomyces cerevisiae? J Mol Evol 2008, 67:621-630.

36. Gach JS, Maurer M, Hahn R, Gasser B, Mattanovich D, Katinger H, Kunert R: High level expression of a promising anti-idiotypic antibody fragment vaccine against HIV-1 in Pichia pastoris. J Biotechnol 2007, 128:735-746.

37. Mashego MR, van Gulik WM, Vinke JL, Heijnen JJ: Critical evaluation of sampling techniques for residual glucose determination in carbonlimited chemostat culture of Saccharomyces cerevisiae. Biotechnol Bioeng 2003, 83:395-399.

38. De Jonge LP, Buijs N, ten Pierick A, Deshmukh A, Zhao Z, Kiel JaKW, Heijnen $J$ J, van Gulik WM: Scale-down of penicillin production in Penicillium chrysogenum. Biotechnol J 2011, 6:944-958.

39. Mashego MR, Wu L, van Dam JC, Ras C, Vinke JL, van Winden W, van Gulik WM, Heijnen JJ: MIRACLE: mass isotopomer ratio analysis of $\mathrm{U}-{ }^{13} \mathrm{C}$-labeled extracts. A new method for accurate quantification of changes in concentrations of intracellular metabolites. Biotechnol Bioeng 2004, 85:620-628.

40. Junker BH, Klukas C, Schreiber F: VANTED: a system for advanced data analysis and visualization in the context of biological networks. BMC Bioinforma 2006, 7:109.

41. Stephanopoulos GN, Aristidou AA, Nielsen J: Material balances and data consistency. In Metabolic Engineering: Principles and Methodologies; 1997.

\section{doi:10.1186/1475-2859-11-83}

Cite this article as: Carnicer et al:: Quantitative metabolomics analysis of amino acid metabolism in recombinant Pichia pastoris under different oxygen availability conditions. Microbial Cell Factories 2012 11:83. 